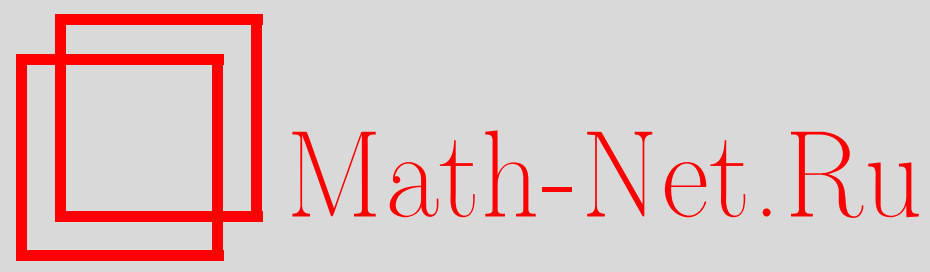

А. Д. Мышкис, Авторегулируемый импульсный точечный подогрев конечной среды, Матем. заметки, 2006, том 79, выпуск 1, 102-106

DOI: https://doi.org/10.4213/mzm2678

Использование Общероссийского математического портала Math-Net.Ru подразумевает, что вы прочитали и согласны с пользовательским соглашением http://www . mathnet.ru/rus/agreement

Параметры загрузки:

IP : 3.89 .185 .249

26 апреля 2023 г., 14:34:37

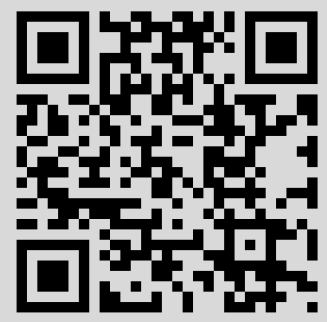




\title{
АВТОРЕГУЛИРУЕМЫЙ ИМПУЛЬСНЫЙ ТОЧЕЧНЫЙ ПОДОГРЕВ КОНЕЧНОЙ СРЕДЫ
}

\author{
А. Д. Мышкис
}

Рассматривается конечная теплопроводящая среда, на границе которой поддерживается нулевая температура, причем одна и та же порция тепла поступает в определенной точке в момент, когда в этой точке температура снижается до заданного уровня. Доказаны существование и единственность, с точностью до произвольного сдвига во времени, периодического режима, имеющего единственный тепловой импульс на каждом периоде. Эффективный алгоритм построения этого режима указан, если средой служит $n$-мерный шар, подогреваемый в его центре, или если средой служит отрезок, подогреваемый в какой-либо его точке.

Библиографияя: 4 названия.

1. Введение. В статье [1] рассмотрен процесс охлаждения стержня, на концах которого поддерживается нулевая температура, причем в моменты, когда общее количество тепла в стержне достигает заданного значения, происходит мгновенная подкачка тепла в стержень по всей его длине. Таким образом, моменты тепловых импульсов не заданы заранее, а определяются самим процессом. В упомянутой статье получены условия существования и единственности в данной ситуации асимптотически устойчивых простых (см. ниже) периодических режимов. Надо отметить, что статья [1] имеет, в основном, методический характер, так как и критерий переключения (общее количество тепла в стержне), и подкачка тепла по всей длине стержня не вполне естественны.

В статье [2] рассматривалась более естественная ситуация. Здесь стержень считался бесконечным, критерием служила температура в какой-либо его фиксированной точке, и считалось, что стержню сообщается определенная порция тепла в этой точке, когда температура в ней достигает определенного значения. Конечно, в такой ситуации периодические режимы невозможны, так что рассматриваются только свойства последовательности моментов импульсов. Асимптотическое выражение для этой последовательности было затем получено в совместной работе китайских математиков Yen-Mei J. Chen, Yunshyong Chow и June Hsieh.

В настоящей статье рассматривается случай, когда среда, в которой распространяется тепло и на границе которой поддерживается нулевая температура, ограниченная, а закон подачи тепловых импульсов такой же, как в [2]. В общей ситуации доказаны существование и единственность, с точностью до произвольного сдвига во времени, простого периодического режима, т.е. имеющего ровно один импульс на каждом своем периоде. Эффективньй алгоритм построения этого режима, указан в двух случаях: когда

Работа выполнена при поддержке Российского фонда фундаментальных исследований, грант № 03-01-00665, и Фонда фундаментальных исследований Министерства транспорта РФ. 
средой служит $n$-мерный шар, подогреваемый в его центре, и когда средой служит отрезок (прямолинейньй стержень), подогреваемый в какой-либо его точке.

2. Общая схема построения периодического решения. Пусть $G \subset \mathbb{R}^{n}, n \geqslant 1$, - ограниченная (открытая) область, $x_{0} \in G$ - произвольная фиксированная точка, в которой будут производиться импульсньй нагрев и измеряться температура. Обозначим через $0<\lambda_{1}<\lambda_{2} \leqslant \lambda_{3} \leqslant \cdots \rightarrow \infty$ последовательность всех собственных значений оператора $U \mapsto-\Delta U$ в $G$ ( $\Delta-$ лапласиан) с краевым условием $\left.U\right|_{\partial G}=0$, а через $\left\{U_{j}\right\}$ - последовательность соответствующих ортонормированных собственных функций. Отметим, что достижение граничного условия понимается, вообще говоря, лишь в обобщенном смысле; для достижения его в обычном смысле достаточно, например, чтобы каждой точки $\partial G$ можно было коснуться конусом, целиком расположенньм вне $G$.

Для произвольной функции $U \in L_{2}(G)$ имеет место разложение $U=\sum_{j=1}^{\infty} c_{j} U_{j}$, где $c_{j}=\int_{G} U U_{j} d G(\forall j \in \mathbb{N})$, а сходимость понимается в смысле $L_{2}(G)$. Если же $U$ - обобщенная функция с носителем, принадлежащим $G$, то справедливы аналогичные формулы, но сходимость понимается в смысле теории обобщенных функций. Отсюда, в частности, вытекает разложение импульсной функции

$$
h \delta\left(x-x_{0}\right)=h \sum_{j=1}^{\infty} U_{j}\left(x_{0}\right) U_{j}(x), \quad h=\text { const }>0,
$$

которая добавляется к распределенной температуре, когда она в точке $x_{0}$ достигает заданного критического значения $u_{c}$.

Так как в рассматриваемой ситуации периодический процесс невозможен без наличия импульсов и так как в силу автономности задачи процесс допускает произвольньй сдвиг во времени, то примем, что в момент $t=0$ температура в точке $x_{0}$ принимает критическое значение: $u(x, 0)=u_{c}$. Записав разложение

$$
u(x, 0)=\sum_{j=1}^{\infty} c_{j} U_{j}(x), \quad x \in G
$$

и учитьвая условие критичности момента времени

$$
\sum_{j=1}^{\infty} c_{j} U_{j}\left(x_{0}\right)=u_{c}
$$

получаем в силу формулы (1)

$$
u\left(x, 0^{+}\right)=\sum_{j=1}^{\infty}\left[c_{j}+h U_{j}\left(x_{0}\right)\right] U_{j}(x) .
$$

Приняв это выражение за начальное распределение температуры, находим закон этого распределения до следующего момента $t=T$ импульсного подогрева:

$$
u(x, t)=\sum_{j=1}^{\infty}\left[c_{j}+h U_{j}\left(x_{0}\right)\right] U_{j}(x) e^{-a \lambda_{j} t}, \quad x \in G, \quad 0<t \leqslant T
$$


где $a$ - коэффициент температуропроводности, который считается заданным.

Если мы хотим получать простое периодическое решение, то полученное выражение должно при $t=T$ совпасть с выражением (2). Отсюда

$$
c_{j}+h U_{j}\left(x_{0}\right) e^{-a \lambda_{j} T}=c_{j},
$$

и потому

$$
c_{j}=\frac{h U_{j}\left(x_{0}\right) e^{-a \lambda_{j} T}}{1-e^{-a \lambda_{j} T}}=\frac{h U_{j}\left(x_{0}\right)}{e^{a \lambda_{j} T}-1} \quad \forall j \in \mathbb{N} .
$$

Подставив это выражение в формулы (3) и (4), приходим к равенствам

$$
\begin{gathered}
h \sum_{j=1}^{\infty} \frac{U_{j}^{2}\left(x_{0}\right)}{e^{a \lambda_{j} T}-1}=u_{c}, \\
u(x, t)=h \sum_{j=1}^{\infty} \frac{U_{j}\left(x_{0}\right) e^{-a \lambda_{j} T}}{1-e^{-a \lambda_{j} T}} U_{j}(x), \quad x \in G, \quad 0<t \leqslant T .
\end{gathered}
$$

Если рассматривать члены ряда $(5)$ как функции $T \in(0, \infty)$, то мы получаем ряд из непрерьвных убывающих функций, равномерно сходящийся на любом интервале $[\tau, \infty)$, $\tau \in(0, \infty)$, причем все эти функции стремятся к бесконечности при $T \rightarrow 0$ и к нулю при $T \rightarrow \infty$. Поэтому и вся левая часть формулы (5) представляет собой непрерывную убывающую функцию $T \in(0, \infty)$, стремящуюся к бесконечности при $T \rightarrow 0$ и к нулю при $T \rightarrow \infty$. Отсюда сразу следует

ТЕОрема 1. При заданных $h$ и $u_{c}$ существует одно и только одно простое периодическое решение поставленной задачи об авторегулируемом импульсном точечном подогреве. Период этого решения определяется с помощью формулы (5), а само решение на своем периоде определяется формулой (6).

Отметим, что формула (5) однозначно определяет любую из трех величин $h, u_{c}$ и $T$, если две другие заданы.

3. Пример I: подогрев шара в его центре. В этом примере в силу теоремы 1 решение задачи о периодическом авторегулируемом импульсном подогреве должно быть центрально-симметричным, т.е. при заданных значениях параметров зависеть только от $r:=|x|$. Поэтому и во всех разложениях должны участвовать только центрально-симметричные собственные функции.

Выражение лапласиана $\Delta$ для центрально-симметричной функции $r \mapsto U(r)$ в $\mathbb{R}^{n}$, $n \geqslant 1$, имеет вид

$$
\Delta U(r)=U^{\prime \prime}(r)+\frac{n-1}{r} U^{\prime}(r),
$$

и потому задача о нахождении центрально-симметричных собственных функций для оператора $-\Delta$ в шаре радиуса $R$ имеет вид

$$
r U^{\prime \prime}(r)+(n-1) U^{\prime}(r)+\lambda r U(r)=0, \quad U(r)=0 .
$$


С помощью подстановки $U(r)=r^{1-n / 2} V(r)$ нетрудно проверить, что совокупность собственных значений и соответствующих нормированных собственных функций такова:

$$
\lambda_{j}=\left(\frac{\mu_{n / 2-1, j}}{R}\right)^{2}, \quad U_{j}(r)=r^{1-n / 2} \frac{J_{n / 2-1}\left(\sqrt{\lambda_{j}} r\right)}{\sqrt{\sigma_{n-1} / 2} R\left|J_{n / 2}\left(\sqrt{\lambda_{j}} R\right)\right|}, \quad j \in \mathbb{N} .
$$

Здесь и далее $J_{p}$ - это функция Бесселя I рода порядка $p, \mu_{j}-$ ее $j$-й положительный нуль, а $\sigma_{p}=2 \pi^{(p+1) / 2} / \Gamma((p+1) / 2)-$ площадь единичной $p$-мерной сфферы $\left(\sigma_{0}=2\right.$, $\sigma_{1}=2 \pi$ и т.д.). Применяемые здесь и далее свойства функции Бесселя можно найти, например, в [3].

Воспользовавшись асимптотическим выражением

$$
J_{p}(z) \sim \frac{1}{\Gamma(p+1)}\left(\frac{z}{2}\right)^{p}, \quad z \rightarrow 0
$$

и формулой для $\sigma_{n-1}$, после простых преобразований получаем предельное выражение

$$
U_{j}(0)=\frac{\lambda_{j}^{(n-2) / 4}}{2^{(n-2) / 2} \pi^{n / 4} R \sqrt{\Gamma(n / 2)}\left|J_{n / 2}\left(\sqrt{\lambda_{j}} R\right)\right|} .
$$

Подставив его в формулу (5), получаем уравнение для определения периода $T$ простого периодического авторегулируемого подогрева шара в его центре с помощью импульса интенсивности $h$

$$
\frac{h}{2^{n-2} \pi^{n / 2} R^{2} \Gamma(n / 2)} \sum_{j=1}^{\infty} \frac{\lambda_{j}^{(n-2)-1}}{J_{n / 2}^{2}\left(\sqrt{\lambda_{j}} R\right)\left(e^{a \lambda_{j} T}-1\right)}=u_{c} .
$$

Закон изменения температуры при этом подогреве можно получать с помощью подстановки выражения (7) в формулу (6).

Отметим, в частности, случай $n=1$, когда, шар превращается в отрезок длины $2 R$, подогреваемьй импульсами из своего центра. Так как

$$
J_{-1 / 2}(x)=\sqrt{\frac{2}{\pi x}} \cos x, \quad J_{1 / 2}(x)=\sqrt{\frac{2}{\pi x}} \sin x
$$

из приведенных вьше обших формул получаем

$$
\mu_{1 / 2, j}=\frac{2 j-1}{2} \pi, \quad \lambda_{j}=\left(\frac{2 j-1}{2 R} \pi\right)^{2}, \quad U_{j}(r)=\frac{1}{\sqrt{R}} \cos \frac{2 j-1}{2 R} \pi r .
$$

Уравнение (8) для определения периода $T$ приобретает вид

$$
\frac{h}{R} \sum_{j=1}^{\infty} \frac{1}{e^{a((2 j-1) \pi /(2 R))^{2} T}-1}=u_{c}
$$


4. Пример II: подогрев прямолинейного стержня. Пусть прямолинейньй отрезок $[-l, l], 0<l<\infty$, подвергается импульсному подогреву в точке $x_{0} \in[0, l)$. Здесь задача о нахождении собственных функций для оператора $-\Delta$ имеет вид

$$
u^{\prime \prime}(x)+\lambda u(x)=0, \quad-l \leqslant x \leqslant l, \quad u( \pm l)=0 .
$$

Поэтому все собственные значения и соответствующие им нормированные собственные функции получаются по формулам

$$
\lambda_{j}=\left(\frac{j \pi}{2 l}\right), \quad U_{j}(x)=\frac{1}{\sqrt{l}} \sin \frac{j \pi(x+l)}{2 l}, \quad j \in \mathbb{N} .
$$

Из общей формулы (5) получаем уравнение для нахождения периода правильных периодических колебаний температуры при авторегулируемом точечном импульсном подогреве

$$
\frac{h}{l} \sum_{j=1}^{\infty} \frac{\sin ^{2}\left[j \pi\left(x_{0}+l\right) /(2 l)\right]}{e^{a[j \pi /(2 l)]^{2} T}-1}=u_{c} .
$$

Нетрудно проверить, что это уравнение для $x_{0}=0$ совпадает с уравнением (9) при $r=l$.

5. Несколько замечаний. Естественно ожидать, что построенные простые периодические режимы асимптотически устойчивы относительно возмущения начальных условий. Однако это пока не доказано, даже при $n=1$ и подогреве в центре стержня. Не исключено, что этот вопрос может быть связан с неким общим утверждением о поведении разрывных автономных систем в бесконечномерном пространстве.

Другое замечание относится к условию простоты периодического режима. Вопрос о существовании и свойствах непростьх периодических режимов пока остается открытым. Возможно, что он связан с проблемой бифуркации циклов (см. [4]) в бесконечномерном пространстве.

Наконец, представляет интерес управление процессом авторегулирования с помощью измерения температуры не в той точке, в которой подается тепловой импульс. Здесь некоторая трудность состоит в немонотонном характере изменения температуры в точке наблюдения между моментами последовательных импульсов.

\section{СПИСОК ЦИТИРОВАННОЙ ЛИТЕРАТУРЫ}

[1] Мышкис А. Д. Процесс теплопроводности с авторегулируемой импульсной поддержкой // Автоматика и телемеханика. 1995. № 2. С. 35-43.

[2] Myshkis A. D. On a recurrently defined sequence // J. Differential Equations Appl. 1997. V. 3. №1. P. 89-91.

[3] Ватсон Г. Н. Теория бесселевых функций. Ч. 1. М.: ИЛ, 1949.

[4] Шарковский А. Н., Майстренко Ю. Л., Романенко Е. Ю. Разностные уравнения и их приложения. Киев: Наукова думка, 1986. 